\title{
Experiments and network model of flow of oil-water emulsion in porous media
}

\author{
Mao Illich Romero and Marcio S. Carvalho* \\ Department of Mechanical Engineering, Pontificia Universidade Catolica do Rio de Janeiro, RJ 22453-900, Brazil \\ Vladimir Alvarado ${ }^{\dagger}$ \\ Department of Chemical and Petroleum Engineering, University of Wyoming, Laramie, Wyoming, USA \\ (Received 10 March 2011; revised manuscript received 12 June 2011; published 10 October 2011)
}

\begin{abstract}
Transport of emulsions in porous media is relevant to several subsurface applications. Many enhanced oil recovery (EOR) processes lead to emulsion formation and as a result conformance originating in the flow of a dispersed phase may arise. In some EOR processes, emulsion is injected directly as a mobility control agent. Modeling the flow of emulsion in porous media is extremely challenging due to the complex nature of the associated flows and numerous interfaces. The descriptions based on effective viscosity are not valid when the drop size is of the same order of magnitude as the pore-throat characteristic length scale. An accurate model of emulsion flow through porous media should describe this local change in mobility. The available filtration models do not take into account the variation of the straining and capturing rates with the local capillary number. In this work, we present experiments of emulsion flow through sandstone cores of different permeability and a first step on a capillary network model that uses experimentally determined pore-level constitutive relationships between flow rate and pressure drop in constricted capillaries to obtain representative macroscopic flow behavior emerging from microscopic emulsion flow at the pore level. A parametric analysis is conducted to study the effect of the permeability and dispersed phase droplet size on the flow response to emulsion flooding in porous media. The network model predictions qualitatively describe the oil-water emulsion flow behavior observed in the experiments.
\end{abstract}

DOI: 10.1103/PhysRevE.84.046305

PACS number(s): 47.56.+r, 47.55.D-, 47.57.Bc, 47.61.Jd

\section{INTRODUCTION}

Oil-water emulsion injection has been found to play an important role in enhanced-oil recovery (EOR) processes. Emulsions can be purposely injected to reduce water production and increase sweep efficiency, acting as a mobility control agent. The potential of emulsions for EOR has been shown in early field tests. McAuliffe [1] presented results in which injecting slugs of oil-in-water emulsion into water injection wells led to an increase in the volumetric sweep efficiency and oil production. To study the mechanisms responsible for the improved oil recovery, emulsion injection in rock samples have been carried out by several researchers. McAuliffe [2] compared water flooding to a dilute emulsion flooding. They observed a delay in the water breakthrough time, indicating a more uniform sweep, and also an increase in the volume of oil produced in the case of emulsion flooding. Emulsion injection to improve sweep efficiency in fractured formation has also been tested [3]. Higher oil recovery was obtained even in cores with longitudinal fracture. Sarma et al. [4] discussed mechanisms for improved oil recovery in heavy oils by injecting water-oil emulsions containing a solvent. They indicate that the process was controlled by the trapping of emulsion droplets and oil viscosity reduction associated with the mixing of oil and solvent. Seright and Liang [5] discussed the efficiency of mobility control agents, including emulsions and foams, and indicate the lack of complete understanding of emulsion mobility control.

\footnotetext{
*msc@puc-rio.br

†valvarad@uwyo.edu
}

Similar improvement in sweep efficiency and volume of oil recovery observed in other EOR processes, such as alkalinesurfactant [6-8] and alkaline-surfactant-polymer [9] flooding, can also be explained by the action of emulsion flow at the pore scale. Emulsions naturally occur as a consequence of decreased interfacial tension in chemical flooding. Low-ionic strength aqueous solutions used for low salinity water and polymer flooding stabilize these naturally occurring emulsions [10,11]. A similar sweep efficiency improvement arises in supercritical $\mathrm{CO}_{2}$ injection in conjunction with surfactant for mobility control or conformance foams [12]. In fact, these foams are in essence supercritical $\mathrm{CO}_{2}$ emulsions rather than the most commonly understood dry foams [13].

The full understanding and design of improved oil recovery processes based on emulsion flooding relies on better understanding the flow of emulsions through porous media and the associated displacement mechanisms. Several experimental analyses of flow of emulsion through porous media have shown that oil drops of the emulsion can block pore throats, which in turn leads to local permeability reduction $[1,14,15]$. Not only do the drops larger than the pore throats reduce the flow, but smaller drops are also captured in crevices and several small drops may cause the same effect as a large drop [16].

Modeling the flow of emulsion in porous media is extremely challenging. The available descriptions based on effective viscosity are not valid when the drop size is of the same order of magnitude as the pore throats. Filtration models have been used to describe the flow of stable, dilute emulsion through porous media $[17,18]$. The model was tested against experimental data on transient permeability, obtaining a good agreement. However, the proposed filtration model does not describe an important aspect of emulsion flow in porous media: 
the permeability reduction caused by the oil drops is more pronounced at smaller differential pressure or low capillary number. Moreover, the parameters associated with the model have to be obtained by macroscopic flow measurements and new measurements would be necessary for each different porous media. Here, we seek a macroscopic flow description that is based on the physics of emulsion flow at the pore scale.

The experiments reported by Cobos et al. [19] on the flow of emulsions through a single cylindrical microcapillary with a throat show that the effect of the dispersed phase diameter on the flow behavior is a strong function of the capillary number when the drops are larger than the constriction diameter. If the capillary number is above a critical value, the deformation of the drop as it passes through the constriction does not change the pressure gradient of the flow. This indicates that the desired mobility control can be achieved at a certain distance from the injection well, since the pressure gradient in radial flow falls as the fluid moves away from the source (well). Again, the design of the emulsion that yields the desired mobility control at a given location in the reservoir, as defined by the local capillary number, is only possible after a more complete understanding of the flow of emulsions through porous media has been attained.

In this research, we studied the flow of emulsions through porous media by experiments and numerical simulation. A capillary network model was developed to obtain upscaled Darcy-level response by using pore-level flow description. The model is based on the flow rate-pressure drop relationship of emulsion flow through a converging-diverging circular quartz capillary with a tube diameter of $200 \mu \mathrm{m}$ and a throat diameter of $50 \mu \mathrm{m}$ [19]. The use of an empirical constitutive relationship based on a limited set of data of the flow through a circular constricted capillary is intended to capture the fundamental physics at the pore scale, not to describe quantitatively the macroscopic flow behavior. However, the main mechanism of pore-throat blockage as a function of capillary number and drop diameter to pore throat diameter ratio should capture the essence of the mobility control mechanism. The experiments consisted of injecting oil-water emulsion with known drop size distribution through sandstone cores with two different permeability values to analyze the effect of drop to pore throat diameter ratio on the steady state pressure drop-flow rate relationship. Experimental results of mobility reduction related to emulsion injection were qualitatively compared with equivalent results obtained through network simulations to verify that the model captures the main elements of the flow.

\section{EXPERIMENTAL ANALYSIS}

\section{A. Experimental setup and materials}

The experimental setup used in the analysis is sketched in Fig. 1. It consisted of a microgear pump (Waters 515 HPLC), transfer vessels, a core holder, and a precision balance. Two pressure transducers (Validyne) were installed to measure the injection pressure and the confining pressure. The injection of either water or emulsion was controlled by opening and closing valves.

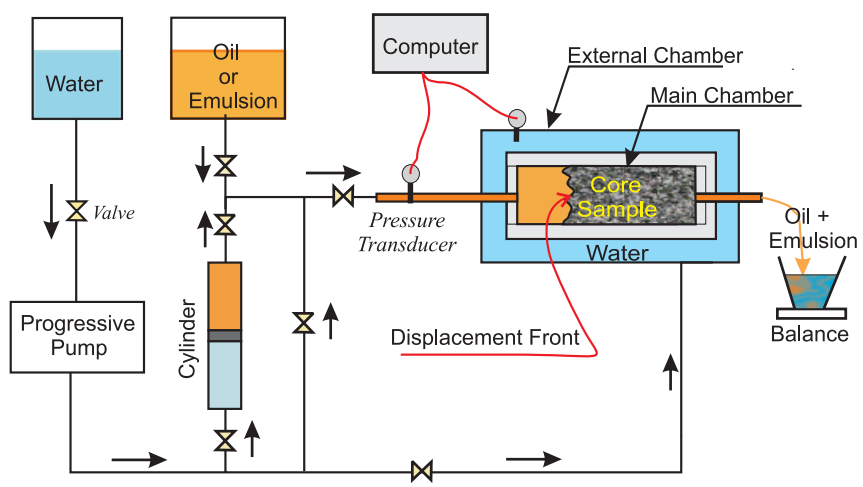

FIG. 1. (Color online) Sketch of experimental setup for emulsion flow through a sandstone core.

The concentration of the dispersed phase of the oil-water emulsion used in the experiments was $10 \%$. The dispersed phase was a mineral oil (Shell Tivela 460) with a viscosity of $\mu_{d}=950 \mathrm{cP}$ and density of $\rho_{d}=949 \mathrm{~kg} / \mathrm{m}^{3}$ at ambient conditions. The continuous phase was a solution of $70 \%$ glycerin and $30 \%$ distilled water with a viscosity of $\mu_{c}=$ $22.5 \mathrm{cP}$ and density of $\rho_{c}=1177 \mathrm{~kg} / \mathrm{m}^{3}$. Glycerin was added to increase the viscosity of the continuous aqueous phase and consequently delay the gravitational segregation of the emulsion in the transfer cylinders during the experiments. Sodium dodecyl sulfate at a concentration of $6.9 \mathrm{~g} / 1$, or approximately three times the critical micelle concentration (CMC), was added to the continuous phase.

The emulsion was prepared using a UltraTurax homogenizer (IKA-Werke 20) at a rotation speed of $24000 \mathrm{rpm}$ for $30 \mathrm{~s}$. The drop size distribution was measured using a Mastersizer 2000 (Malvern Instruments). An example of the drop size distribution, represented in terms of number of drops, is shown in Fig. 2. All drops were smaller than $40 \mu \mathrm{m}$ and

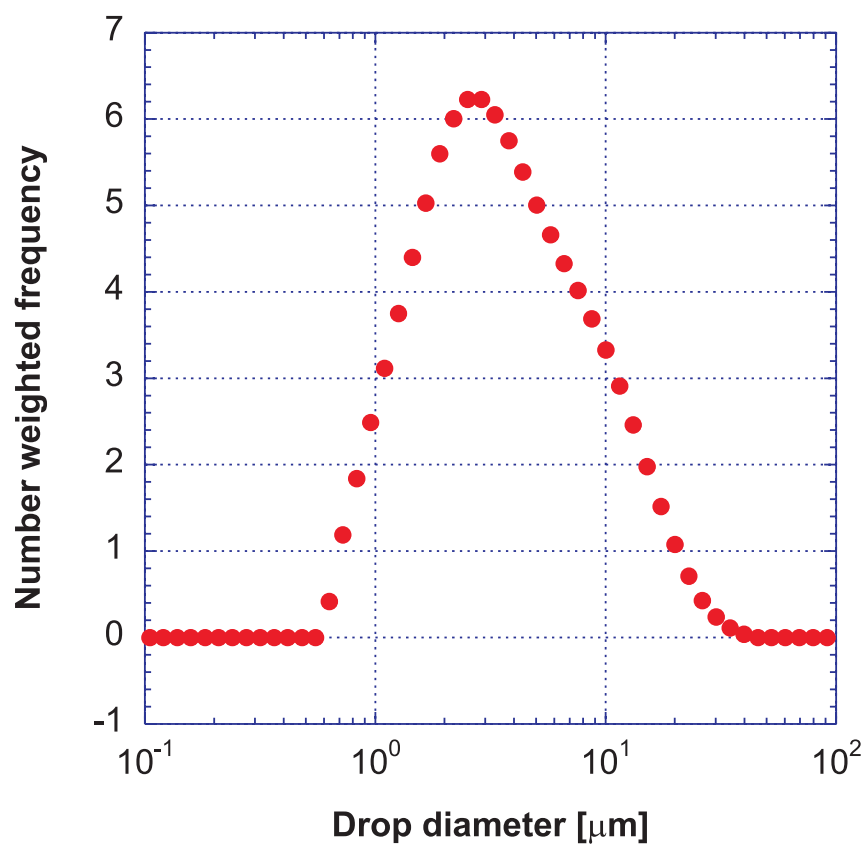

FIG. 2. (Color online) Drop size distribution of the oil-water emulsion used in the experimental analysis. 
TABLE I. Properties of sandstone cores.

\begin{tabular}{lcccc}
\hline \hline Core & $\begin{array}{c}\text { Length } \\
(\mathrm{mm})\end{array}$ & $\begin{array}{c}\text { Area } \\
\left(\mathrm{cm}^{2}\right)\end{array}$ & $\begin{array}{c}\text { Porosity } \\
(\%)\end{array}$ & $\begin{array}{c}\text { Permeability } \\
(\mathrm{mD})\end{array}$ \\
\hline $\mathrm{L}$ & 76.8 & 11.1 & 27.3 & 106 \\
$\mathrm{H}$ & 49 & 10.9 & 31.9 & 655 \\
\hline \hline
\end{tabular}

the mean drop diameter was approximately $d \approx 6 \mu \mathrm{m}$. The emulsion viscosity as a function of the shear rate was measured on a rotational rheometer (Anton Paar Physica MCR301) with a Couette fixture. The viscosity was apparently constant and equal to $\mu_{e}=26 \mathrm{cP}$. Because of the low oil volumetric fraction, the effect of the dispersed phase on the emulsion viscosity was small.

Two sandstone cores, from Botucatu (Brazil), were used as porous media. They were initially saturated with distilled water in order to measure pore volume and porosity. The cores were then inserted in a rubber sleeve and inside the core holder unit. The permeability of each sample was determined by measuring the volumetric flow rate of water as a function of the pressure drop across the cores. The results together with length $L$ and cross section area $A$ of each core are shown in Table I. The lower and higher permeability cores are labeled $L$ and $H$, respectively.

The experimental setup was used in a parametric analysis to determine the effect of capillary number (flow rate) and permeability in emulsion flow through porous media. The results were used as basis for a qualitative validation of the proposed network model, described in the following section.

\section{CAPILLARY NETWORK MODEL}

A complete geometrical characterization of the pore space of the sandstone samples used in the experiments, needed to create a capillary network, was not available. Instead, we have used the network geometry developed by Oren et al. [20] and used by Piri and co-workers [21,22]. The network represents a $3 \times 3 \times 3 \mathrm{~mm}^{3}$ cube of a Berea sandstone sample. It is made of 26146 capillaries and 12349 pore bodies. The elements of the network (pore bodies and capillaries) had triangular, quadrilateral, and circular cross sections, with the vast majority (approximately 92\%) being triangular. The coordination number of the pores varies from 1 to 19 , with an average coordination number equal to 4.18 . The porosity of the network is $\phi_{N}=24 \%$ and its permeability is equal to $K_{N}=2560 \mathrm{mD}$. For the simulations presented here, $K_{N}$ was adjusted to the values observed in the experiments $K$ by multiplying the geometry (length and radius) of every element of the network by the geometric scale factor [23]

$$
\delta=\sqrt{\frac{K}{K_{N}}} .
$$

Although the network geometry did not represent the sandstone sample used in the experimental analysis, it can be used to verify that the proposed model is able to capture the main physics of emulsion flow though porous materials. In a network model, the flow rate $q_{i j}$ through a capillary element that connects pores $i$ and $j$ is written as a function of the pressure difference $\Delta p_{i j}=p_{i}-p_{j}$,

$$
q_{i j} \equiv g_{i j} \frac{p_{i}-p_{j}}{l_{i j}} .
$$

For a single-phase flow, the conductance $g_{i j}$ is fully determined by the geometry of the capillary cross section and the liquid viscosity. For complex fluids, the conductance is not as simply determined. The constitutive equation that relates pressure drop to flow rate in each capillary of the network, i.e., that defines the capillary conductance $g_{i j}$, was developed based on the experimental results for emulsion flow through a single constricted microcapillary [19], as described in the following subsection.

\section{A. Flow of emulsion through a single constricted capillary: Constitutive relationship}

The constitutive flow rate-pressure drop relationship in each capillary of the network was derived empirically by constructing an algebraic relation that extrapolates the experimental results of Cobos et al. [19] and is able to describe the basics of the flow of emulsions through constricted microchannels. Oil-in-water emulsions were injected into a quartz circular capillary tube possessing two straight sections joined by a constriction, which mimicked a pore throat. In the experiments, the pressure drop along the capillary tube ends was recorded as a function of time at each flow rate until a steady state was reached. The effect of the dispersed phase on the pressure drop-flow rate relation was represented in terms of a mobility reduction or scale factor $f$, defined as the ratio of the pressure drop for the continuous phase flow $\Delta P_{c}$ to that of the emulsion flow $\Delta P_{e}$ at the same flow rate,

$$
f=\frac{\Delta P_{c}}{\Delta P_{e}} .
$$

Therefore, in a capillary with radius $r_{c}$ subjected to a pressure difference of $\Delta P$, the flow rate $Q$ is evaluated as

$$
Q=f \frac{r_{c}^{2}}{8} \frac{\pi r_{c}^{2}}{\mu_{c}} \frac{\Delta P}{L} .
$$

The physical meaning of this factor can be interpreted as a dimensionless mobility correction that originates from the blockage of a pore throat that reduces the liquid mobility. The experiments show that when drops are much smaller than the constriction radius, $f$ is independent of the capillary number and approximately equal to one. When drops are of the same size or larger than the throat radius, $f$ is a strong function of the capillary number. At high enough capillary number, $f$ approaches a constant value. The extra pressure required to deform the drop is much smaller than the pressure drop associated with viscous effects; the mobility reduction is achieved simply because a lower viscosity liquid (water) was substituted by a higher viscosity liquid (oil). At low enough capillary number, the capillary pressured related to the drop deformation is of the same order or larger than the viscous pressure drop and the value of $f$ becomes much smaller than 1. The transition between the plateau and the strong capillary number dependent region occurs at a critical capillary number, 
$\mathrm{Ca}^{*}$, which in principle is a function of the liquid properties and geometry of the capillary.

In summary, the resulting constitutive relationship for each capillary of the network, expressed through the mobility correction $f$, should reflect the following function behavior:

(1) For drop radius $r_{e}$ to pore-throat radius $r_{c}$ ratio, denoted as $\alpha \equiv r_{e} / r_{c}$, below a critical value $\alpha^{*}$, the flow is not affected by the presence of the dispersed phase. The mobility reduction factor is $f=1$.

(2) For $\alpha>\alpha^{*}$, the mobility reduction factor is a function of the drop to capillary diameter ratio and capillary number: $f=f(\alpha, \mathrm{Ca})$. At capillary number above a critical value $\mathrm{Ca}^{*}$, capillary forces are not relevant and the mobility factor is only a function of $\alpha$.

We sought a simple and convenient functional form for the $f$ factor that satisfied the aforementioned conditions. For $\alpha<\alpha^{*}$,

$$
f=1 \text {. }
$$

For $\alpha>\alpha^{*}$,

$$
f= \begin{cases}e^{-k_{A}\left(\alpha / \alpha^{*}-1\right)}\left(\frac{\mathrm{Ca}}{\left.\mathrm{Ca}^{*}\right)^{k_{B}\left(\alpha / \alpha^{*}-1\right)},}, \mathrm{Ca}<\mathrm{Ca}^{*},\right. \\ e^{-k_{A}\left(\alpha / \alpha^{*}-1\right)}, & \mathrm{Ca}>\mathrm{Ca}^{*} .\end{cases}
$$

The empirical parameters that describe the flow through a single capillary are (i) $\alpha^{*}$, the critical drop to pore throat diameter ratio above which the flow is affected by the presence of the dispersed phase, (ii) $\mathrm{Ca}^{*}$, the critical capillary number below which the dispersed drop deformation contributes for the reduced mobility, and (iii) $k_{A}$ and (iv) $k_{B}$ are fitting parameters that describe how the mobility reduction factor $f$ varies with drop diameter and capillary number. Figure 3 shows the mobility factor $f$ as a function of $\mathrm{Ca}$ for different values of $\alpha$ measured by Cobos et al. (points in the plot). The

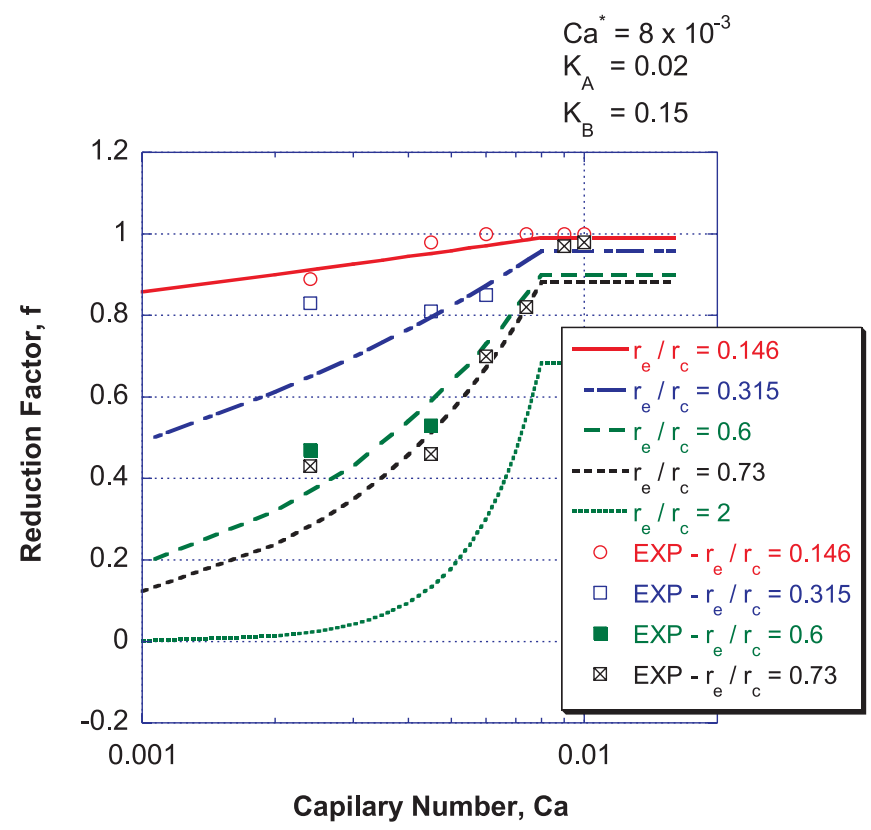

FIG. 3. (Color online) Mobility reduction factor for the flow through a single constricted capillary. Lines represent the calculation using the empirical relation proposed here; symbols represent experimental data of Cobos et al. [19]. data were used to estimate the model parameters: $\mathrm{Ca}^{*}=0.008$, $\alpha^{*}=0.1$, and $k_{A}=0.02$ and $k_{B}=0.15$. The continuous lines on Fig. 3 represent the mobility reduction factor given by Eq. (5), which, as shown, is able to describe the flow behavior. The coefficients of the constitutive behavior of the flow in each capillary of the network should be determined based on experiments of emulsions through microchannels that are representative of the pore throat geometry.

A few simplifying assumptions are introduced into the model. The capillary used in the experiments by Cobos et al. [19] were circular, whereas most of the elements in the network are of triangular cross section. Triangular cross section enables the flow of the continuous phase close to the corner regions, which may quantitatively change the pore level mobility control factor. However, the basic mechanism, described by the proposed functional form of $f$, of mobility control is associated with the dispersed phase deformation as it flows through pore throats and it is independent of the cross section shape. Another simplifying assumption is that one drop radius is used to calculate the mobility reduction factor. This turns out to be an acceptable simplification, if one realizes that the larger drops dominate the pressure drop behavior and their numerical abundance in the emulsion is small, although their volumetric fraction is large, so the dominant drop size fraction is relatively small. Moreover, we consider that the dispersed phase is present in every element of the network. In reality, emulsion drops may partially block different paths of the pore space leading to a nonuniform distribution of dispersed phase throughout the porous media. However, at steady state, the model should become more adequate.

\section{B. Solution method}

The conductivity of each capillary is a function of the geometry of the capillary, emulsion drop diameter, the continuous phase viscosity, and the local capillary number (capillary flow rate), making the relation nonlinear. The

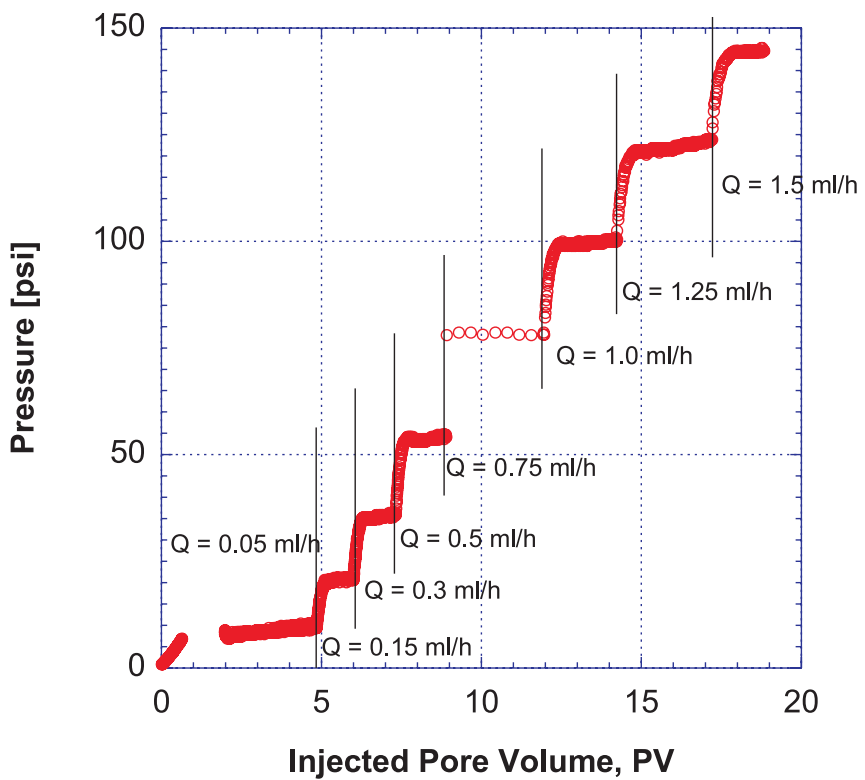

FIG. 4. (Color online) Evolution of the measured inlet pressure as the flow rate was changed in steps. The results are for core $L$. 
pressure at each node of the network is determined by applying mass conservation equation at each pore element. The resulting set of nonlinear equations was solved iteratively. The pressure at each node was first determined assuming the flow of the continuous phase alone. In this case, the conductivity of each element is not a function of the flow rate and the system of equation is linear. The computed pressure at each node and flow rate through each capillary was used as the initial guess for the nonlinear problem.

The model was used in a parametric analysis to determine the effect of capillary number (flow rate) and emulsion drop diameter in emulsion flow through a porous media. The ability
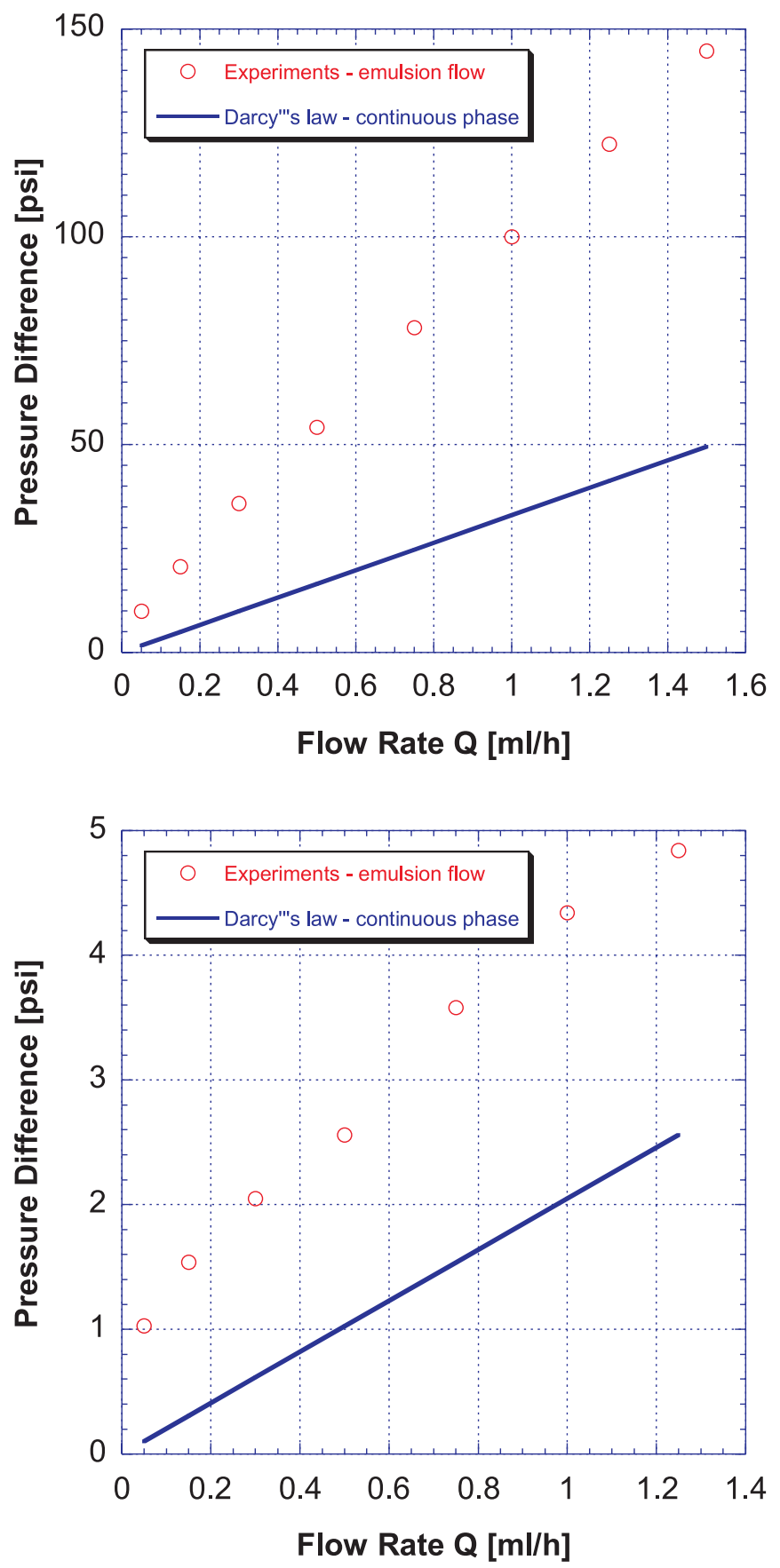

FIG. 5. (Color online) Inlet pressure as a function of flow rate for emulsion flow. (a) Results for core L; (b) results for core $\mathrm{H}$. of the model to describe qualitatively different aspects of emulsion flow through porous materials is discussed.

\section{RESULTS}

Emulsion was injected into the sandstone cores, initially saturated with distilled water, at low flow rate, $Q=0.05$ $\mathrm{ml} / \mathrm{min}$, which corresponds to a capillary number $\mathrm{Ca} \equiv$ $\mu_{c} \bar{V} / \sigma=8 \times 10^{-6}$. The capillary number is defined in terms of the continuous phase viscosity $\mu_{c}$, the oil-water interfacial tension $\sigma$, and the Darcy velocity $\bar{V} \equiv Q / \pi R^{2}$. The inlet pressure was registered as a function of time until steady state was obtained. At this point, the inlet pressure and the imposed flow rate were recorded. The flow rate was raised in small steps to a maximum value of $Q=1.5 \mathrm{ml} / \mathrm{min}$ for the lower permeability core $\mathrm{L}$ and $Q=3.0 \mathrm{ml} / \mathrm{min}$ for the higher permeability core $\mathrm{H}$. The maximum capillary number achieved was $\mathrm{Ca} \approx 1.05 \times 10^{-4}$ for core $\mathrm{H}$ and $\mathrm{Ca} \approx 1.25 \times 10^{-4}$ for core L. It is important to notice that for both cores, the maximum capillary number is below the critical capillary number $\mathrm{Ca}^{*}=0.008$ above which the mobility reduction factor $f$ of the flow through the microcapillary is not a function of the capillary number.

At each flow rate, the inlet pressure data was recorded after each new steady state was reached. Figure 4 shows the evolution of inlet pressure as a function of time for the entire range of flow rate explored. The results are for core L. At each flow rate, steady state was reached after approximately 1 pore volume.

The averaged steady state pressure drop as a function of flow rate in the experiments for both cores is shown in Fig. 5. The plots also show the pressure drop for the flow of the continuous phase alone evaluated using Darcy's law. Although the emulsion viscosity was virtually constant with respect to shear rate, i.e., the emulsion presented a Newtonian behavior,

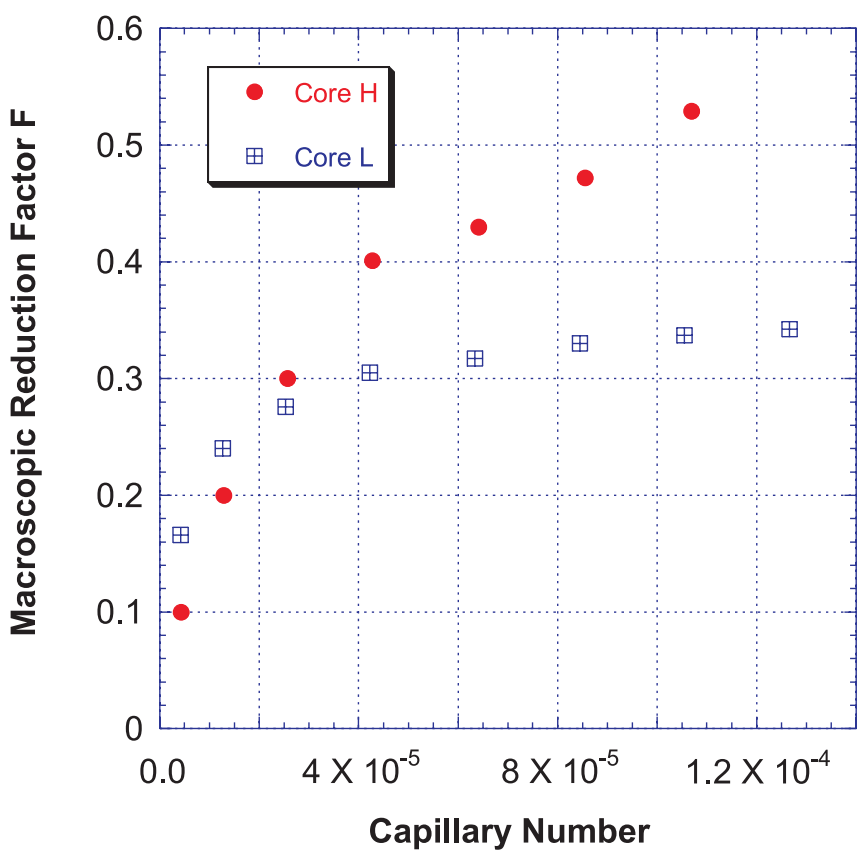

FIG. 6. (Color online) Macroscopic reduction factor $F$ evaluated from experimental analysis of flow of emulsion through porous media. 
the nonlinear behavior of the response is clear, mainly at low flow rate.

The effect of the dispersed phase on the flow through porous media can be characterized by defining a macroscopic mobility reduction factor $F$, such that

$$
q=\frac{Q}{A}=F \frac{K}{\mu_{c}} \frac{\Delta p_{e}}{L} .
$$

This represents the ratio between the pressure drop obtained in the flow of the continuous phase alone, $\Delta p_{c}$, to the pressure drop measured in the flow of the emulsion, $\Delta p_{e}$,

$$
F=\frac{\Delta p_{c}}{\Delta p_{e}}=\frac{\mu_{c}}{K} \frac{Q}{A} \frac{L}{\Delta p_{e}}
$$

The data in Fig. 5 were used to evaluate the macroscopic mobility reduction factor $F$ at different conditions, which is shown in Fig. 6. For the higher permeability core $H$, the maximum value of the macroscopic mobility reduction factor was $F \approx 0.5$. The effect of the dispersed phase is more pronounced at lower capillary numbers.

The results obtained with the lower permeability core $L$ show that the dispersed phase has a stronger effect on the flow. The maximum reduction factor is approximately $F \approx 0.35$. In this core, the pore throat diameters are smaller than in core $\mathrm{H}$ and the drop diameter to pore throat diameter ratio is much

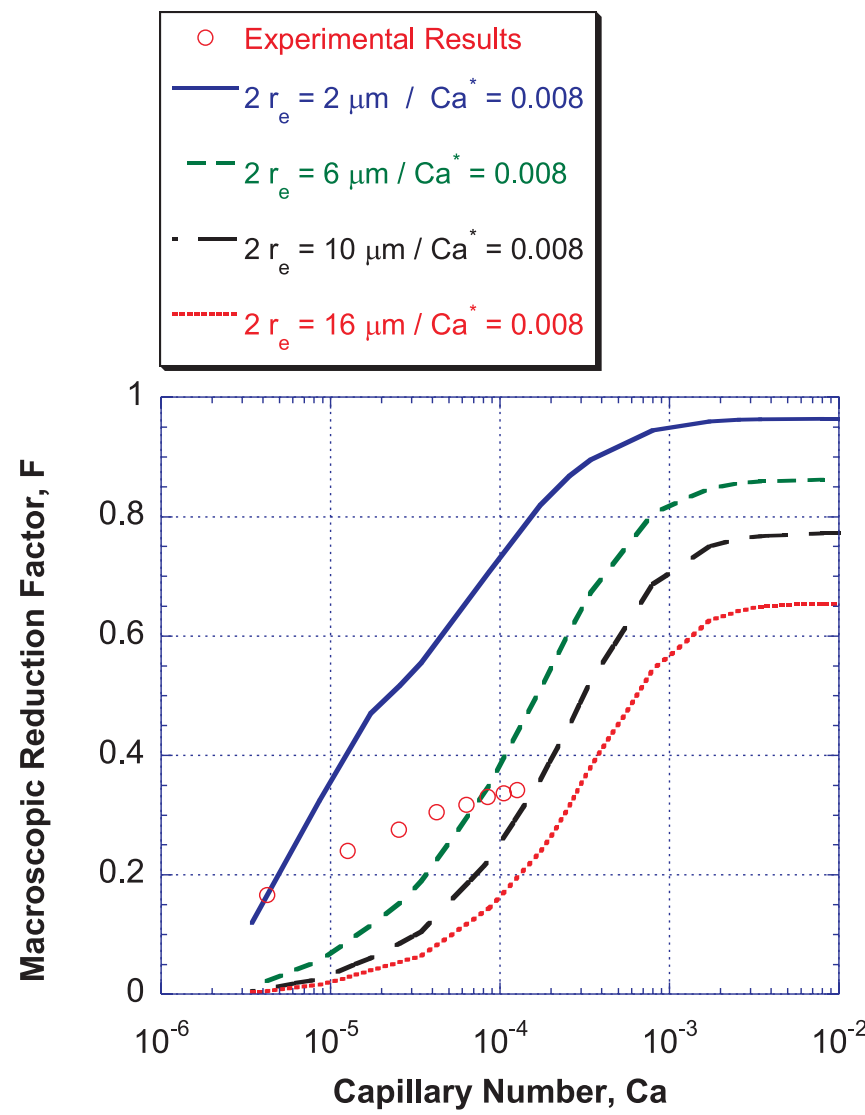

FIG. 7. (Color online) Network model predictions of the macroscopic reduction factor $F$ at low permeability at different values of emulsion drop radius (parameter $\alpha$ ) and critical capillary number (parameter $\mathrm{Ca}^{*}$ ). The experimental results presented in Fig. 6 are also shown. larger. At high capillary numbers, $F$ is almost constant; the higher pressure drop at a fixed flow rate is not related to the extra capillary pressure needed to deform the drop tip but to the simple substitution of a lower viscosity liquid (continuous phase) by a higher viscosity liquid (dispersed phase) in the narrow pore throats [19]. At low capillary numbers, e.g., $\mathrm{Ca}<$ $4 \times 10^{-5}$, the reduction factor falls sharply as the capillary number decreases. In this case, the mobility reduction is associated with the extra pressure needed to deform the drops as they pass through the narrow pore throats.

Figure 7 replots the experimental data shown in Fig. 6 together with predictions obtained from the network model adjusted to have the same permeability of core L, i.e., $K=$ $106 \mathrm{mD}$. The parameters for the pore scale flow are those used to fit the experimental data of flow through a singular constricted microcapillary, $\mathrm{Ca}^{*}=0.008, \alpha^{*}=0.1$, and $k_{A}=$ 0.02 and $k_{B}=0.15$. Predictions were obtained at different drop diameters, e.g., $2 r_{e}=2,6,10$, and $16 \mu \mathrm{m}$. The effect of drop diameter is clear in the plot. Emulsion with large drops leads to a smaller macroscopic reduction factor $F$. With the smaller drops and high capillary number, the effect of the dispersed phase is weak, i.e., $F \approx 0.95$. With the larger drops, the dispersed phase occupies most of the cross section area of the pore throats leading to a higher pressure drop at a fixed flow rate. For $2 r_{e}=16 \mu \mathrm{m}, F \approx 0.65$ at high capillary number.

The predictions for drop diameter of $2 r_{e}=6$ and $10 \mu \mathrm{m}$ are in the same order of magnitude as the experimental

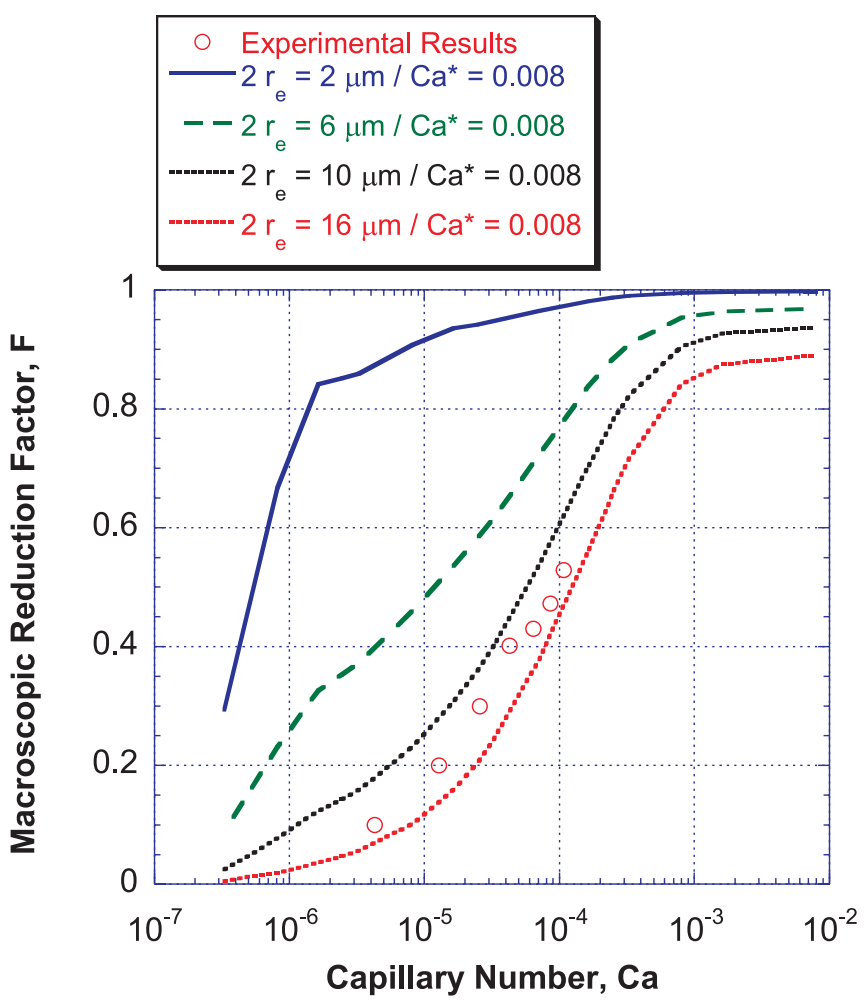

FIG. 8. (Color online) Network model predictions of the macroscopic reduction factor $F$ at high permeability at different values of emulsion drop radius (parameter $\alpha$ ) and critical capillary number (parameter $\mathrm{Ca}^{*}$ ). The experimental results presented in Fig. 6 are also shown. 
measurements, which were performed with an emulsion that had a mean drop diameter of approximately $6 \mu \mathrm{m}$. However, the sensitivity to capillary number in the experiment was weaker.

The network model predictions for the higher permeability core $\mathrm{H}$ and the corresponding experimental measurements are shown in Fig. 8. In this case, the prediction obtained with $2 r_{e}=6$ and $10 \mu \mathrm{m}$ matches the experimental data well. It is important to notice that the effect of the permeability is clear. For emulsion with the same drop size, the macroscopic reduction factor $F$ is higher in the higher permeability network, in which the pore throats are larger.

The network model presented here is able to reproduce qualitatively the experimental data for flow of emulsions through porous materials of different permeabilities. The main reasons for the quantitative discrepancy, mainly at lower permeability, between the model and experiments are as follows:

(1) The function used to describe the reduction factor in each capillary of the network is based on experiments performed in circular capillaries and on data points from a limited set of experimental conditions and may not fully describe the full physics of the pore scale flow at a broad range of flow conditions.

(2) The model considers a monodispersed emulsion.

(3) The model considers the concentration of the emulsion to be the same throughout the network, which may be approximate at best.
(4) The network used was not based on the sandstone sample used in the experiments and may have a different pore body and pore throat radii distribution.

Despite these quantitative discrepancies, the network model is able to describe the dependency of the flow on the local capillary number that has been observed experimentally not only in this work but also in the experiments by others $[2,15]$.

\section{CLOSING REMARKS}

We have successfully developed a network model that represents the flow of emulsions as an effective single phase fluid flow by incorporating empirical data from single-capillary tube results. The network model captures qualitatively the correct behavior of emulsion flow through sandstone samples, which demonstrates the ability of the model to capture the main physical mechanisms associated with flow of oil-inwater emulsions. Moreover, the network model correctly produces a qualitatively correct dependence of the mobility reduction factor $f$ as a function of the capillary number. Further investigation is necessary to obtain pore-scale flow rate-pressure drop data to improve model predictions.

\section{ACKNOWLEDGMENTS}

This research was funded by grants from the Brazilian Research Council (CNPq) and Petrobras. We would like to acknowledge the Enhanced Oil Recovery Institute at the University of Wyoming for additional financial assistance.
[1] C. D. McAuliffe, J. Pet. Technol. 25, 727 (1973).

[2] C. D. McAuliffe, J. Pet. Technol. 25, 721 (1973).

[3] L. Romero, J. L. Zirit, A. Marín, F. Rojas, J. L. Mogollón, E. Manrique, and F. Paz, Proceedings of the SPE/DOE Tenth Symposium on Improved Oil Recovery, Tulsa, OK, USA 35461 , 611 (1996).

[4] H. K. Sarma, B. B. Maini, and K. Jha, J. Can. Pet. Technol. 37, 55 (1998).

[5] R. S. Seright and J. Liang, Proceedings of the European Formation Damage Conference, The Hague, The Netherlands, SPE 30120, 431 (1995).

[6] M. Arhuoma, D. Yang, M. Dong, H. Li, and R. Idem, Energy Fuels 23, 5995 (2009).

[7] M. Arhuoma, M. Z. Dong, and D. Y. Yang, Ind. Eng. Chem. Res. 48, 7092 (2009).

[8] J. Wang, M. Dong, and M. Arhuoma, J. Can. Pet. Technol. 49, 51 (2010).

[9] P. Shen, J. Wang, S. Yuan, T. Zhong, and X. Jia, SPE J. 14, 237 (2009).

[10] M. Moradi, V. Alvarado, and S. Huzurbazar, Energy Fuels 25, 260 (2011).

[11] X. Wang, A. Brandvik, and V. Alvarado, Energy Fuels 24, 6359 (2010).
[12] R. F. Li, W. Yan, S. Liu, G. J. Hirasaki, and C. A. Miller, SPE J. 15, 934 (2010).

[13] F. Rouyer, O. Pitois, E. Lorenceau, and N. Louvet, Phys. Fluids 22, 043302 (2010).

[14] D. A. Alvarado and S. S. Marsden Jr., SPE J. 19, 369 (1979).

[15] J. A. Hofman and H. N. Stein, Colloids Surf. 61, 317 (1991).

[16] H. Soo and C. J. Radke, Ind. Eng. Chem. Fundam. 23, 342 (1984).

[17] H. Soo and C. J. Radke, Chem. Eng. Sci. 41, 263 (1986).

[18] H. Soo, M. C. Williams, and C. J. Radke, Chem. Eng. Sci. 41, 273 (1986).

[19] S. Cobos, M. S. Carvalho, and V. Alvarado, Int. J. Multiphase Flow 35, 507 (2009).

[20] P. E. Oren, S. Bakke, and O. J. Arntzen, SPE J. 3, 324 (1998).

[21] M. Piri and M. J. Blunt, Phys. Rev. E 71, 26302 (2005).

[22] Available for download from the Imperial College web site [http://www3.imperial.ac.uk].

[23] X. Lopez, P. H. Valantine, and M. J. Blunt, J. Colloid Interface Sci. 264, 256 (2003). 